BULLETIN OF THE

AMERICAN MATHEMATICAL SOCIETY

Volume 77, Number 2, March 1971

\title{
THE STRUCTURE OF $\omega$-REGULAR SEMIGROUPS
}

\section{BY JANET AULT AND MARIO PETRICH}

Communicated by R. S. Pierce, July 20, 1970

1. Finding the complete structure of regular semigroups of a certain class has succeeded only when sufficiently strong conditions on idempotents and/or ideals have been imposed. On the one hand, there is the theorem of Rees [7], giving the structure of completely 0 -simple semigroups, and its successive generalizations to primitive regular semigroups [2], and $J$ - and $J_{1}$-regular semigroups [4]. On the other hand, with very different restrictions, Reilly [8] has determined the structure of bisimple $\omega$-semigroups, Kočin [1] of inverse simple $\omega$-semigroups, Munn [5] of inverse $\omega$-semigroups.

An $\omega$-chain with zero is a poset $\left\{e_{i} \mid i \geqq 0\right\} \cup 0$ with $e_{i}>e_{j}$ if $i<j$, and $0<e_{i}$ for all $i, j$. We call a regular semigroup $S \omega$-regular if $S$ has a zero and the poset of its idempotents is an orthogonal sum [2] of $\omega$-chains with zero. We announce here the complete determination of the structure of such semigroups, including various special cases thereof, and briefly mention their isomorphisms.

2. An $\omega$-regular semigroup can be uniquely written as an orthogonal sum of $\omega$-regular prime (i.e., with 0 a prime ideal) semigroups. This reduces the problems of structure and isomorphism to $\omega$-regular prime semigroups. We distinguish three cases: (i) 0 -simple, (ii) prime with a proper 0 -minimal ideal, (iii) prime without a 0 -minimal ideal. Case (i) is the most difficult (and interesting) and includes a variety of special cases some of which reduce to those constructed by Reilly [8], Kočin [1], and Munn [5], [6].

3. Let $A$ be a nonempty set, $d$ be a positive integer, $V$ be a semigroup which is a chain of $d$ groups $G_{0}>G_{1}>\cdots>G_{d-1}$, and $\sigma$ be a homomorphism of $V$ into $G_{0}$. Let $w: A \rightarrow\{0,1, \cdots, d-1\}$ be any function, denoted by $w: \alpha \rightarrow w_{\alpha}$. For $\alpha \in A, 0 \leqq i, j<d$, define $\langle\alpha, i\rangle$ by

$$
\langle\alpha, i\rangle \equiv w_{\alpha}+i(\bmod d), \quad 0 \leqq\langle\alpha, i\rangle<d,
$$

and define $[i, \alpha, j]$ to satisfy

$$
[i, \alpha, j] d=(i-j)-(\langle\alpha, i\rangle-\langle\alpha, j\rangle) .
$$

AMS 1970 subject classifications. Primary 20M10.

Key words and phrases. Regular semigroups, $\omega$-regular, $\omega$-semigroups, 0-simple, 0-bisimple, prime semigroups, orthogonal sum, Brandt semigroups, primitive inverse semigroups, 0-restricted homomorphisms, semilattice, tree, Brandt tree, rooted Brandt tree. 
Construction I. On the set

$$
S=\{(\alpha, m, g, n, \beta) \mid \alpha, \beta \in A, m, n \geqq 0, g \in V\} \cup 0,
$$

define a multiplication by: for $g_{i} \in G_{i}, g_{j} \in G_{j}, v=n-s-[i, \beta, j]$,

$$
\begin{array}{r}
\left(\alpha, m, g_{i}, n, \beta\right)\left(\beta, s, g_{j}, t, \gamma\right)=\left(\alpha, m-[i, \alpha, j]-v,\left(g_{i} \sigma^{-v}\right) g_{j}, t, \gamma\right) \\
\text { if } v<0, \text { or } v=0, i \leqq j \\
\left(\alpha, m, g_{i}, n, \beta\right)\left(\beta, s, g_{j}, t, \gamma\right)=\left(\alpha, m, g_{i}\left(g_{j} \sigma^{v}\right), t+[i, \gamma, j]+v, \gamma\right) \\
\text { if } v>0, \text { or } v=0, i>j ;
\end{array}
$$

and all other products are equal to 0 . The set $S$ with this multiplication will be denoted by $\mathcal{O}(A, w ; V, \sigma)$.

Construction II. On the set

$$
S^{\prime}=\left\{(\alpha, m, g, n, \beta) \mid \alpha, \beta \in A, m-w_{\alpha} \equiv n-w_{\beta} \equiv i(\bmod d), g \in G_{i}\right\} \cup 0,
$$

define a multiplication by: for $g_{i} \in G_{i}, g_{j} \in G_{j}, v=n^{\prime}-s^{\prime}-[i, \beta, j]$, where $n=n^{\prime} d+n^{\prime \prime}, s=s^{\prime} d+s^{\prime \prime}, 0 \leqq n^{\prime \prime}, s^{\prime \prime}<d$,

$$
\begin{aligned}
\left(\alpha, m, g_{i}, n, \beta\right)\left(\beta, s, g_{j}, t, \gamma\right) & =\left(\alpha, m+s-n,\left(g_{i} \sigma^{-v}\right) g_{j}, t, \gamma\right) \text { if } n \leqq s \\
\left(\alpha, m, g_{i}, n, \beta\right)\left(\beta, s, g_{j}, t, \gamma\right) & =\left(\alpha, m, g_{i}\left(g_{j} \sigma^{v}\right), t+n-s, \gamma\right) \text { if } n>s
\end{aligned}
$$

and all other products are equal to 0 . The set $S^{\prime}$ with this multiplication will be denoted by $\mathcal{O}[A, w ; V, \sigma]$.

The following is our fundamental result.

THEOREM 1. For a groupoid $S$, the following statements are equivalent.

(i) $S$ is a 0 -simple $\omega$-regular semigroup;

(ii) $S$ is isomorphic to $\mathcal{O}(A, w ; V, \sigma)$;

(iii) $S$ is isomorphic to $\mathcal{O}[A, w ; V, \sigma]$.

The proof of "(i) $\Rightarrow$ (ii)" consists of "introducing coordinates" into various $\mathcal{L}$ - and $\mathcal{R}$-classes and of constructing the homomorphism $\sigma$; it is quite long and is broken into a sequence of lemmas. For "(ii) $\Rightarrow$ (iii)" one finds a suitable isomorphism, while "(iii) $\Rightarrow(\mathrm{i})$ " consists of a verification of the defining properties of a 0 -simple $\omega$-regular semigroup.

Define the top of $S$ in the theorem by

$J(S)=\{a \in S \mid e \& a, a \propto f$ for some maximal idempotents $e, f\} \cup 0$.

Then $J(S)$ is a primitive inverse semigroup. It follows from the proof that we can always suppose that $w_{\alpha}=0$ for some $\alpha \in A$. Call $S$ balanced if any two maximal idempotents of $S$ are D-equivalent.

THEOREM 2. The following conditions on a 0 -simple $\omega$-regular semigroup $S$ are equivalent. 
(i) $S$ is balanced;

(ii) $S$ admits a representation as in Theorem 1 with $w_{\alpha}=0$ for all $\alpha \in A$;

(iii) $J(S)$ is a Brandt semigroup;

(iv) $S$ is isomorphic to a Rees matrix semigroup $\operatorname{Tl}^{0}(K ; A, A ; \Delta)$ over a simple inverse $\omega$-semigroup $K, \Delta$ is the identity matrix.

The structure of the semigroup $K$ in Theorem 2 was determined by Kočin [1] and Munn [5], the Rees matrix semigroups over bisimple inverse semigroups were studied in [3] (for the 0 -simple case in the theorem, cf. [3, Corollary 5.7] and [6, Theorem 4.2]). Various other special cases include: 0 -bisimple, combinatorial, balanced, and combinations thereof.

4. For the remaining cases, we will need the following.

Construction III. Let $Y$ be a tree semilattice satisfying one of the two conditions: (1) $Y$ has a zero $\zeta$ and all elements of $Y$ are of finite height, (2) $Y$ has no zero and is of locally finite length. To every nonzero element $\alpha$ of $Y$ associate a Brandt semigroup $S_{\alpha}$, suppose that the family $\left\{S_{\alpha}\right\}$ is pairwise disjoint, and that a homomorphism $\phi_{\alpha}: S_{\alpha} \rightarrow S_{\bar{\alpha}}$ is given, where $\bar{\alpha}$ is the unique element of $Y$ covered by $\alpha$, with the properties:

(i) $S_{\alpha} \phi_{\alpha} \cap S_{\beta} \phi_{\beta}=0$ if $\bar{\alpha}=\bar{\beta}$;

(ii) for every infinite ascending chain $\alpha_{1}<\alpha_{2}<\ldots$ in $Y$ and every $a \in S_{\alpha_{1}}^{*}$, there exists $\alpha_{k}$ such that $a \notin S_{\alpha_{k}} \phi_{\alpha_{k}} \phi_{\alpha_{k-1}} \cdots \phi_{\alpha_{2}}$. Let $\psi_{\alpha, \alpha}$ be the identity mapping on $S_{\alpha}$, and for $\alpha>\beta$, let $\psi_{\alpha, \beta}=\phi_{\alpha} \phi_{\alpha_{1}} \cdots \phi_{\alpha_{n}}$ where $\alpha \succ \alpha_{1} \succ \cdots \succ \alpha_{n} \succ \beta$. Let $S=\left(\cup_{\alpha \in Y \backslash \zeta}\left(S_{\alpha} \backslash 0_{\alpha}\right)\right) \cup_{0}$ where $\zeta$ is the zero of $Y$ (if $Y$ has one), and 0 is an element not contained in any $S_{\alpha}$, and on $S$ define the multiplication $*$ by

$a * b=\left(a \psi_{\alpha, \alpha \beta}\right)\left(b \psi_{\beta, \alpha \beta}\right)$ if $\alpha \beta \neq \zeta \quad$ and $\quad\left(a \psi_{\alpha, \alpha \beta}\right)\left(b \psi_{\beta, \alpha \beta}\right) \neq 0_{\alpha \beta}$ in $S_{\alpha \beta}$,

and all other products are equal to 0 . The set $S$ with this multiplication will be called a Brandt tree if $Y$ has a zero and a rooted Brandt tree otherwise.

TheOREM 3. A semigroup $S$ is prime w-regular and has a proper 0minimal ideal if and only if $S$ is an ideal extension of a 0 -simple $\omega$ regular semigroup $I$ by a Brandt tree $T$ determined by a 0-restricted homomorphism of $T$ into the top of $I$.

Such a homomorphism is completely determined by its restriction to the socle $\mathfrak{S}(T)$ of $T$, so all such homomorphisms are given by 0 restricted homomorphisms of $\mathfrak{S}(T)$ into $\mathfrak{J}(I)$, both of which are primitive inverse semigroups, and are easy to find explicitly. 
TheOREM 4. A groupoid $S$ is a prime w-regular semigroup without 0-minimal ideals if and only if $S$ is a rooted Brandt tree.

5. The semigroups $\mathcal{\Theta}(A, w ; V, \sigma)$ and $\Theta[A, w ; V, \sigma]$ do not seem to admit a neat isomorphism theorem except in special cases. In the balanced case, using Theorem $2,[3,4.1]$, and $[1$, Theorem 4], we derive a satisfactory isomorphism theorem. A direct proof does the same in the case these semigroups are combinatorial. Isomorphisms of the semigroups in Construction III are similar to those in $[4$, Théorème 3.1], while isomorphisms of the semigroups in Theorem 3 can be expressed by isomorphisms of $I$ and $T$ satisfying a commutative diagram.

\section{REFERENCES}

1. B. P. Kočin, The structure of inverse ideally-simple w-semigroups, Vestnik Leningrad. Univ. 23 (1968), no. 7, 41-50. (Russian) MR 37 \#2881.

2. G. Lallement and M. Petrich, Décompositions I-matricielles d'un demi-groupe, J. Math. Pures Appl. (9) 45 (1966), 67-117. MR 33 \#5765.

3. - A generalization of the Rees theorem in semigroups, Acta Sci. Math. (Szeged) 30 (1969), 113-132. MR 39 \#2899.

4. - Structure d'une classe de demi-groupes réguliers, J. Math. Pures Appl. 48 (1969), 345-397.

5. W. D. Munn, Regular w-semigroups, Glasgow Math. J. 9 (1968), 46-66. MR $37 \# 5316$.

6. —_, 0-bisimple inverse semigroups, J. Algebra 15 (1970), 570-588.

7. D. Rees, On semi-groups, Proc. Cambridge Philos. Soc. 36 (1940), 387-400. MR 2,127.

8. N. R. Reilly, Bisimple w-semigroups, Proc. Glasgow Math. Assoc. 7 (1966), 160-167. MR 32 \#7665.

The Pennsylvania State University, University Park, Pennsylvania 16802 